\title{
Cosmic magnetic fields in the Sun: Current Outstanding Problems (Invited Review)
}

\author{
Eric Priest \\ Mathematics Institute, University of St Andrews, \\ North Haugh, St Andrews, KY16 9SS, UK \\ email: eric@mcs.st-and.ac.uk
}

\begin{abstract}
In the Sun there has been much progress towards answering fundamental problems with profound implications for the behaviour of cosmic magnetic fields in other stars. A review is given here of such problems, including identifying some of the outstanding questions that remain. In the solar interior, the main dynamo operates at the base of the convection zone, but its details have not been identified. In the solar surface, recent observations have revealed many new and surprising properties of magnetic fields, but understanding the key processes of flux emergence, fragmentation, merging and cancellation is rudimentary. Sunspots have until very recently been an enigma. In the atmosphere, there are many new ideas for coronal heating and solar wind acceleration, but the mechanisms have not yet been pinned down. Also, the detailed mechanisms for solar flares and coronal mass ejections remain controversial. In future, new generations of space and ground-based measurements and computational modelling should enable a definitive physical understanding of these puzzles.
\end{abstract}

Keywords. Magnetic fields, MHD, sun: corona, sunspots, sun: photosphere.

\section{Introduction}

There has been a revolution in solar physics over the past 10 years, with many advances that have far-reaching implications for other stars, where similar processes are operating but under different parameter regimes. Major progress has been made on fundamental questions about the nature of the Sun, such as the dynamo generation of its magnetic field, sunspot structure, coronal heating, solar wind acceleration, solar flare and coronal mass ejection origin, but as yet no definitive answers have been given. Here I give a brief overview of this new Sun, referring to the review talks that follow and leaving the listeners to make their own connections to similar phenomena on other stars.

These advances have arisen from a combination of ground-based observations, theoretical and computational modelling, and especially space observations from the following satellites: the Yohkoh mission (1992-2002) which revealed the dynamic nature of the corona; the SoHO mission $(1995-\ldots)$ which viewed the interior and atmosphere; the TRACE satellite (1998-2010) which showed the fine-scale nature of the corona; RHESSI $(2002-\ldots)$ which has been studying high-energy processes in solar flares. More recently, the Stereo spacecraft $(2006-\ldots)$ has built up stereoscopic images of coronal mass ejections from two locations, and the Hinode satellite $(2006-\ldots)$ has studied the connections between photosphere and corona, as described in the talk by Saku Tsuneta (Fig. 1a).

In future, we expect to learn much from the Sunrise mission (see talk by Sami Solanki) and from the Solar Dynamics Observatory (launched in February, 2010), which includes EVE (extreme ultraviolet variability experiment), HMI (heliospheric and magnetic 

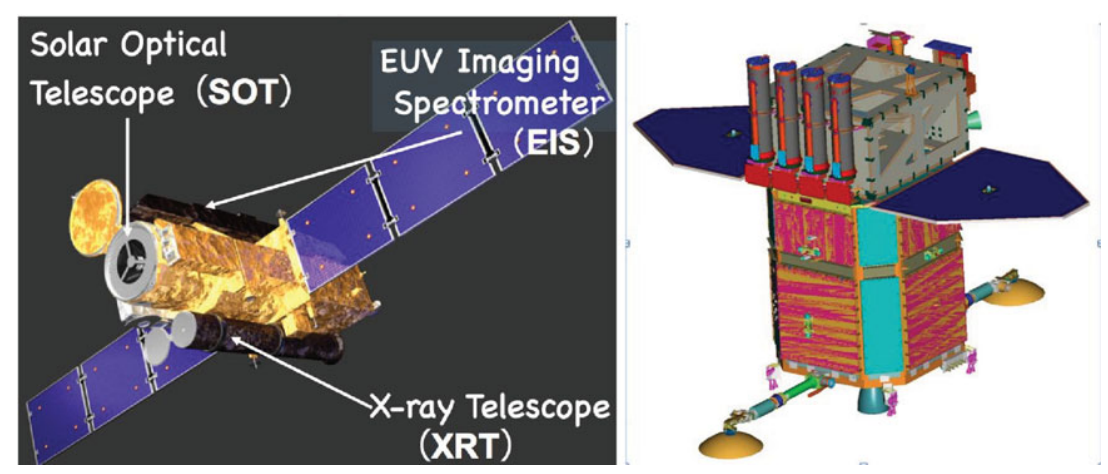

Figure 1. The Hinode satellite (left) and Solar Dynamics Observatory (right).

imager) and AIA (atmospheric imaging assembly). AIA in particular is a super-TRACE instrument, which has the same spatial resolution as TRACE but for the full disc and with images at 8 temperatures (from 20,000 K to $20 \mathrm{MK}$ ) every 10 seconds (Fig. 1b).

So what is the current status of understanding and what are the main questions about the solar interior, photosphere and corona?

\section{The Solar interior}

The solar interior has been opened up to study by the advent of helioseismology, whereby the properties of global and local oscillations have been observed by groundbased networks (GONG and Bison) as well as the MDI instrument on SoHO. The deduced internal temperature structure agrees with the standard model to within $1 \%$, but the internal rotation structure has been a big surprise. At the solar surface, the equator rotates more rapidly than the poles, and so the internal rotation was expected to be constant on cylinders with the dynamo generated throughout the convection zone.

However, the seismology result was that rotation is instead constant on cones in the convection zone and possesses a strong shear layer at the base of the convection zone, termed the tachocline. It is now believed that the main solar dynamo responsible for active regions and sunspots is generated at the tachocline, although the details are uncertain.

The main questions about the solar interior, therefore, are:

What is the detailed internal flow structure, especially near the poles and below the convection zone, as well as the deep-seated nature of the meridional flow?

For dynamo theory, there is uncertainty about the validity of mean-field theory and the alpha effect. Also, is the main dynamo generated at the tachocline or is it generated by a Babcock-Leighton effect at the solar surface? Furthermore, the nature of the connection between the tachocline and the radiative interior and convection zone is unclear. (See the talk by Arnab Choudhuri for clarification of these issues.)

Robust, reliable predictions of the solar cycle are also needed. The current solar minimum, the lowest for 200 years, was a big surprise to most of us (except for Matt Penn, who will be talking later). When and how large will the next solar maximum be? 

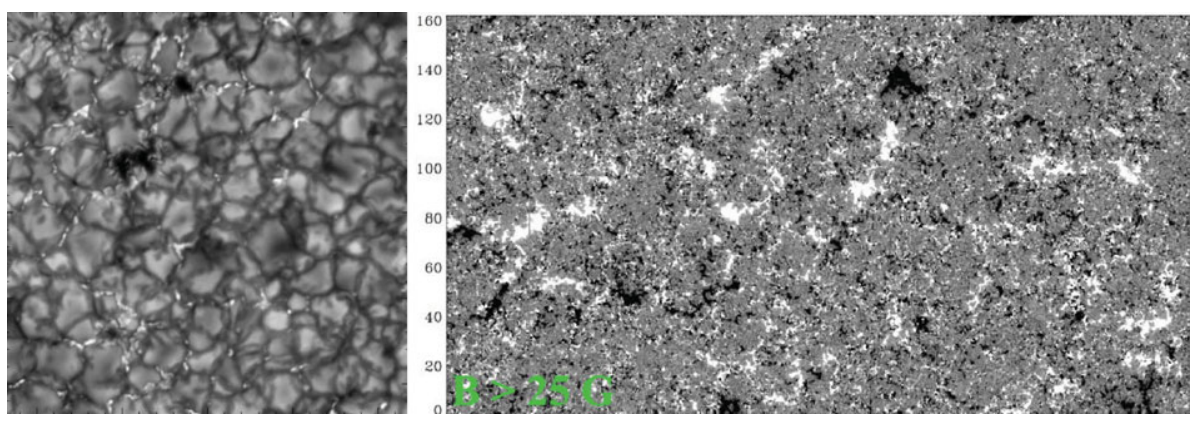

Figure 2. (a) A close-up of the photosphere from the Swedish Solar Telescope (courtesy M. Carlsson and the Swedish Academy of Sciences). (b) The line-of-sight photospheric magnetic field from Hinode (courtesy B. Lites).
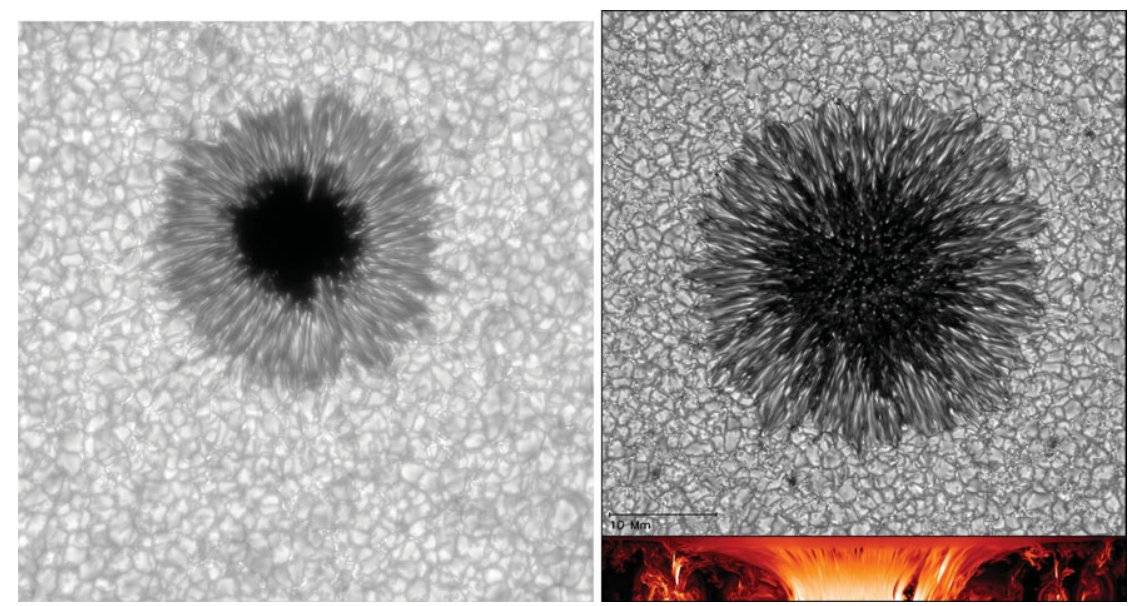

Figure 3. Images of sunspots from: (a) the Swedish Solar Telescope (courtesy L. Rouppe van Der Voordt and Swedish Academy of Sciences); (b) numerical computation (courtesy M. Rempel).

\section{The Photosphere}

The standard picture was until recently that outside active regions the photospheric magnetic field is vertical and concentrated at the edges of supergranule cells (of width 15$30 \mathrm{Mm}$ by comparison with the solar radius of $700 \mathrm{Mm}$ ). Magnetic flux emerged mainly as ephemeral active regions in the supergranule cell interiors and then fragmented as it was carried to the cell boundaries. It then moved along the cell boundaries and either merged with like-polarity elements or cancelled with those of opposite polarity. Such flux was reprocessed every 14 hours (Hagenaar et al. 2003).

However, observations of the photosphere from the Swedish Solar Telescope on La Palma has revealed bright points at the edges of granules that represent intense magnetic flux concentrations (Figure 2a). Furthermore, the Hinode satellite has measured the line-of-site field at low thresholds and deduced that there is a huge amount of complex vertical magnetic flux in the interior of superganules in the boundaries between individual granules (Figure 2b). In addition, a big surprise was its discovery of ubiquitous horizontal fields in the photosphere on the edges of granules (see talk by Saku Tsuneta). 
Turning to sunspots, again the Swedish Solar Telescope has produced remarkable images with 0.03 arcsec pixels, which show fine-scale granular behaviour and incredibly small scales in the penumbra (Figure 3a). Furthermore, the Hinode satellite has revealed that the penumbral magnetic field varies strongly on a small scale and has a comb-like structure, with low-lying field lines closing back down just outside the sunspot and more oblique field lines arching much higher in the atmosphere.

Until recently, the nature of the penumbra was a mystery that several quite different models tried to explain. However, a new breakthrough has come from a series of numerical computations by Matthias Rempel of magnetoconvection, with remarkably realistic-looking images of model sunspots (Figure $3 \mathrm{~b}$ ). Previously, the nature of, say umbral dots, penumbral filaments, Evershed flow and moat flow had been explained by separate models, but Rempel et al. (2009) give a unified explanation for them all in terms of the natural effects of convection in an inclined magnetic field (see talk by Rempel).

The main questions for the Quiet Sun are: how much flux is inside supergranules and how much is at the edge? What is the effect of the fine-scale structure on coronal heating? What is the ultimate size of photospheric flux tubes? For the latter, I would estimate a size of $30 \mathrm{~km}$, based on the photospheric magnetic diffusivity.

For sunspots, what is the nature of the outer penumbra and the subphotospheric structure and are the penumbral field lines held down by granular flux pumping?

These are tackled in the talks by Carsten Denker, Laurent Gizon, Kioshi Ichimoto, Valentin Martinez, Matthias Rempel and Rolf Schlichenmaier.

\section{The Corona}

\subsection{Chromospheric and coronal magnetic fields}

Firstly, how can we measure solar magnetic fields reliably? Talks by José Carlos del Toro and Hongqi Zhang will address this in the photosphere and chromosphere. In the corona it is much harder and so we need to rely for many purposes on extrapolations from the photosheric field. Potential extrapolations are usually very poor: - for example, a comparison of coronal holes and regions of open magnetic fields in global potential extrapolations is highly disappointing. Nevertheless, nonlinear force-free extrapolations both of local and global fields are much more helpful (see talk by Thomas Wiegelmann).

The global coronal magnetic field evolves in response to the emergence of new flux, magnetic diffusion, meridional flow and differential rotation. These have been incorporated into a model which impressively predicts the location of most quiescent prominences outside active regions (Mackay \& van Ballegooijen 2006). It also predicts the eruptions of many coronal mass ejections (see talk by Duncan Mackay).

\subsection{Coronal Heating}

The nature of coronal heating remains a mystery. Close et al. (2004) constructed magnetic field lines in the Quiet Sun from SoHO MDI magnetograms and found that each photospheric source connects on average to 8 others. Most of them close down close to the surface and a few reach large heights. More surprisingly, when they followed the motions of the photospheric sources and recalculated the connectivity, they found that the time for all the field lines on the Quiet Sun to reconnect is only 1.5 hours. In other words, there is an incredible amount of reconnection going on continually in the corona and this is probably responsible for its heating. 


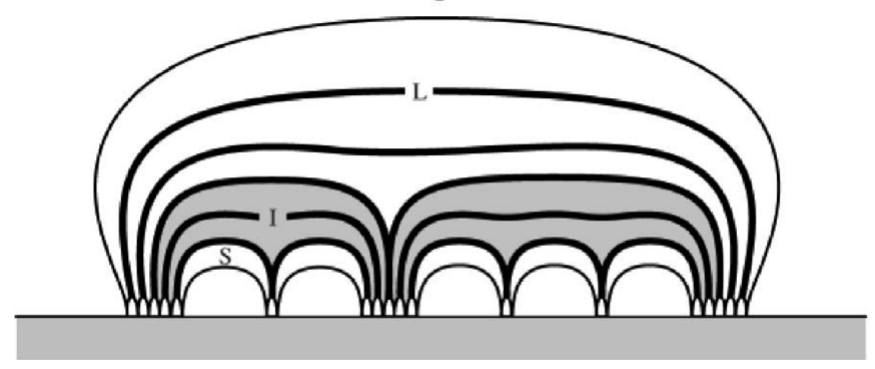

Figure 4. The Coronal Tectonics Model.

The topology of coronal fields is highly complex, but an invaluable method for estimating the topology is to calculate the skeleton of the field. In two dimensions, a plane is separated up into different regions by separatrix curves that intersect at an X-type null point. In three dimensions, a volume is separated into topologically different regions by separatrix surfaces, which meet in a separator - a special field line that usually begins and ends at a null point. The skeleton then consists of a web of separatrix surfaces. Parnell et al. (2009) have developed sophisticated techniques to calculate such a skeleton for any magnetic structure, whether produced in a numerical experiment or arising as a result of a photospheric extrapolation.

The Coronal Tectonics Model (Priest et al. 2002) was developed in order to take account of the magnetic carpet and to refine Parker's braiding model for coronal heating (Figure 4). Each coronal loop that is currently observed in practice goes down through the solar surface in many tiny sources. Thus, the flux from each source is separated by a separatrix surface. The idea of the model is then that the corona is filled with myriads of current sheets forming and dissipating impulsively at separators and also at QuasiSeparatrix Layers where the mapping gradient is large rather than being infinite (as it is at a separatrix) (see Priest \& Démoulin (1995); Démoulin et al. (1996); Aulanier et al. (2006)).

A key question is how to identify the mechanism or mechanisms that are heating the corona. Toshifumi Shimizu will describe new observations of active regions, and Daniel Gomez will consider the role of turbulence, while Klaus Glasgaard and Guillaume Aulanier will describe heating at null points, separators and quasi-separatrix layers.

\subsection{Solar flares}

By comparing the structure of active regions with the location of solar flares, some authors suggest they occur at separators (Longcope et al. 2007), while others suggest the location is a quasi-separatrix layer (Aulanier et al. 2006).

Four suggestions have been proposed for the cause of the magnetic eruption that produces a coronal mass ejection and two-ribbon solar flare. These are kink instability, magnetic nonequilibrium, torus instability and breakout (Figure 5). A key question is therefore to determine for realistic active regions and flare situations (Figure 6) which of the four possibilities possesses the correct physics (see talk by Guillaume Aulanier). 


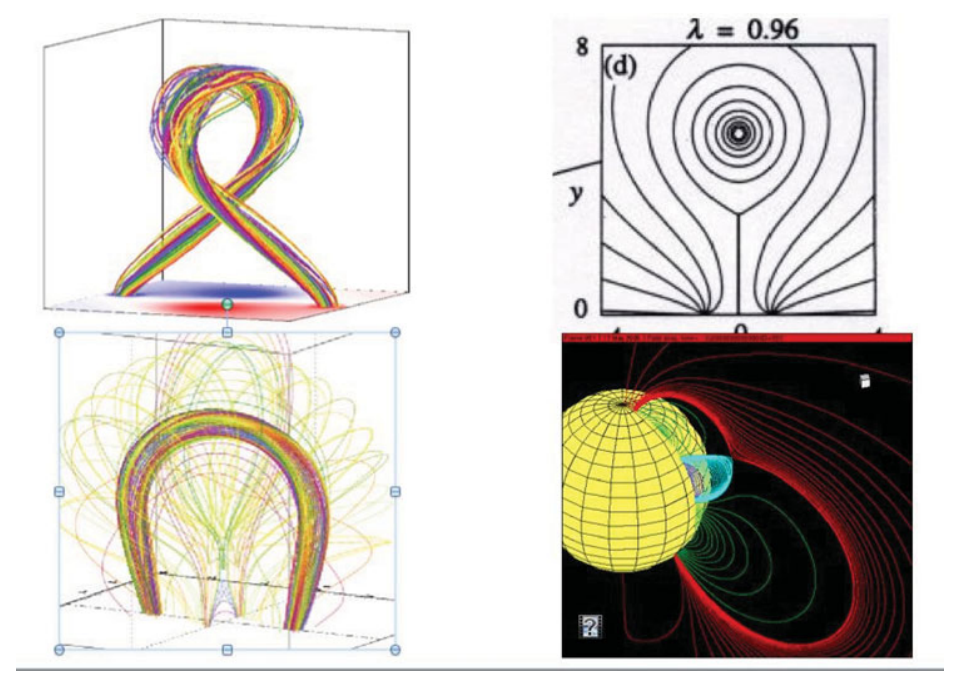

Figure 5. Four possible models for the eruption of coronal magnetic fields in a two-ribbon solar flare or coronal mass ejection.

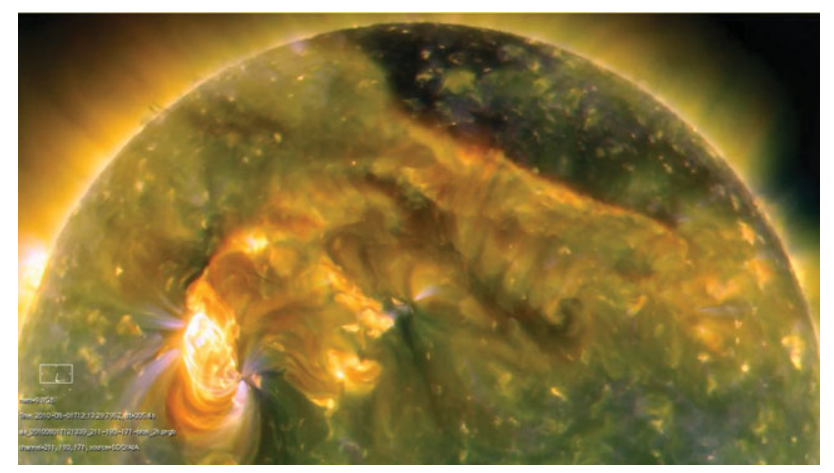

Figure 6. An image from AIA on the Solar Dynamics Observatory of the active solar corona during the occurrence of several solar flares (courtesy K Schrijver).

\section{Conclusions}

Solar physics is currently in a golden age of great vitality as we home in on explanations for many of the fundamental questions in the field. These include the nature of the solar interior and the dynamo process, the behaviour of photospheric magnetic fields and sunspots, the heating of the corona and the origin of solar flares.

In this endeavour computational experiments and space observations provide a key role. It will be fascinating to hear the insights of the array of outstanding speakers that have been assembled for this meeting.

\section{References}

Aulanier, G., Pariat, E., Démoulin, P., \& Devore, C. R. 2006, Solar Phys., 238, 347 Close, R., Parnell, C., Mackay, D. H., \& Priest, E. R. 2004, Solar Phys., 212, 251

Démoulin, P., Henoux, J. C., Priest, E. R., \& Mandrini, C. H. 1996, Astron. Astrophys, 308, 643 Hagenaar, H. J., Schrijver, C. J., \& Title, A. M. 2003, Astrophys. J., 584, 1107

Longcope, D. W., Beveridge, C., Qiu, J., Ravindra, B., Barnes, G., \& Dasso, S. 2007, Solar Phys., 244, 45S 
Mackay, D. H. \& van Ballegooijen, A. A., 2006, Astrophys. J., 641, 577

Parnell, C. E., Haynes, A. L., \& Galsgaard, K., 2009, Journal of Geophysical Research, 115, 2102 Priest, E. R. \& Démoulin, P., 1995, Journal of Geophysical Research, 23, 443

Priest, E. R., Heyvaerts, J., \& Title, A. M., 2002, Astrophys. J., 576, 533

Rempel, M., Schüssler, M., Cameron, R. H., \& Knölker, M., 2009, Science, 325, 171 